\title{
Nanoarchitecturing of Activated Carbon: Facile Strategy for Chemical Functionalization of the Surface of Activated Carbon**
}

\author{
Yi-Jun Xu, Gisela Weinberg, Xi Liu, Olaf Timpe, Robert Schlögl, and Dang Sheng Su* \\ Department of Inorganic Chemistry, Fritz Haber Institute of the Max Planck Society, Faradayweg 4-6, 14195, Berlin \\ (Germany) \\ *Corresponding author: E-mail: dangsheng@fhi-berlin.mpg.de \\ ** This work is carried out in the framework of the EnerChem project of the Max Planck Society, Germany. \\ Supporting Information is available online from Wiley InterScience or from the author.
}

Nanoarchitecturing of carbon nanospheres onto the surface of activated carbon (AC) gives birth to a new composite carbon material that features a hierarchical structure with macro- and nanometer dimensions of the respective carbon components and exhibits a remarkably enhanced adsorption capability for heavymetal ions $\left(\mathrm{CrO}_{4}{ }^{2-}\right.$ and $\left.\mathrm{Fe}^{3+}\right)$ from aqueous solution as compared to AC. Thus, we first propose that nanoarchitecturing of AC can be utilized not only as a flexible method for the synthesis of novel, hybrid, nanostructured composite carbon materials but also as a new and "green-route" strategy for functionalization of the surface of AC in an effective manner. Hence, there is scope for a possible new concept in the functionalization of industrial AC for specific applications.

\section{Introduction}

Nanostructured carbon materials continue to attract considerable interest because of their unique combination of chemical and physical properties, diverse morphology, and numerous potential applications, such as nanoscale engineering and molecular electronics, microfilters, gas sensors, hydrogen-storage devices, and catalysis.[1-3] We mainly focus on the assembly or synthesis of new hybrid nanostructured carbon materials, understanding the characteristics of nanostructured carbon materials at a microscopic level, and their potential applications.[4] Herein, we report a facile and greensynthesis route of novel composite carbon material by nanoarchitecturing of activated carbon under mild conditions. We show that the as-obtained composite carbon material features a hierarchical structure of the respective carbon components on macro- and nanometer scales and, in particular, has a significantly enhanced adsorption capability for heavy-metal ions in aqueous solution as compared to single-activated carbon. The idea underlying this work is the presupposition that a proper combination of macro- and nanometer dimensions in a controlled fashion would lead to novel, hybrid, hierarchically nanostructured composite materials, thereby exhibiting improved or overall the best properties of each component. This Full Paper will describe a simple and green strategy for synthesis of novel composite carbon materials for target application. Thus, we propose for the first time that nanoarchitecturing of activated carbon can be used as not only a flexible method for producing novel composite carbon materials but also a new and green alternative to functionalizing the surface of activated carbon rather than conventional methods,[5] such as the use of environmentally unfavorable reagents, such as strong acids, bases, or designed organic ligands, for modification of the surface of activated carbon.

It is well-known that adsorption on the surface of activated carbon has great potential for the removal of trace amounts of toxic heavy metals in waste water.[6] The adsorption of metal ions from solution on activated carbon is usually dominated by the surface functional groups on the activated carbon, and, consequently, a key aspect in designing suitable activated carbons for metal capture is the control of the surface functionalization chemistry. However, the effective control of this surface chemical functionalization has remained a challenging task when the usual activation techniques are used. This is particularly true when the attainment of activated carbons shows a marked specificity for a particular metal ion species,[5a] which is in great contrast to the well-established methods that are employed to prepare carbon materials possessing optimal properties of surface area and specific pore size distribution.[7,8] Furthermore, note that conventional methods for functionalization of the activated carbon surface involve using environmentally unfavorable reagents, for example, ozone in the gas phase, and strong acids or bases or harmful organic ligands in the solution phase.[5] Hence, there is an urgent need for the development of new strategies or concepts to functionalize the surface of activated carbon 
in an effective manner. Toward this end, we report the nanoarchitecturing of activated carbon, which can be employed as a new, facile, and green strategy for effective functionalization of the surface of activated carbon. This opens the possibility of a new route for functionalization of the surface of activated carbon for specific applications.

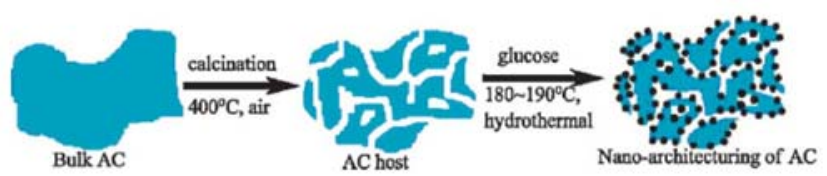

Scheme 1. Nanoarchitecturing of activated carbon by anchoring nanostructured carbon spheres onto the AC host.

\section{Results and Discussion}

The production of this composite carbon material is outlined in Scheme 1. The bulk activated carbon (AC) raw material is made from palm kernel shells, a waste product from palm oil production; this raw material was also used in previous work on the synthesis of a hierarchically structured carbon system with carbon nanofibers (CNFs) nested inside and immobilized onto modified activated carbon (Fe/AC) by a high-temperature chemical vapor deposition (CVD) method.[9] Bulk AC was first calcined at $400{ }^{\circ} \mathrm{C}$ for 4h in air; this step selectively removes the soft parts of the biopolymer and boosts the AC pores, thus, transforming bulk AC aggregates into a macroporous scaffold (see Supporting Information, Fig. S1). This AC host is then utilized for growing nanostructured carbon spheres by means of a glucose solution as precursor in an appropriately controlled hydrothermal treatment.

a)

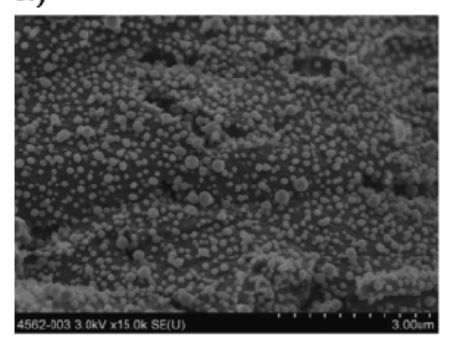

b)

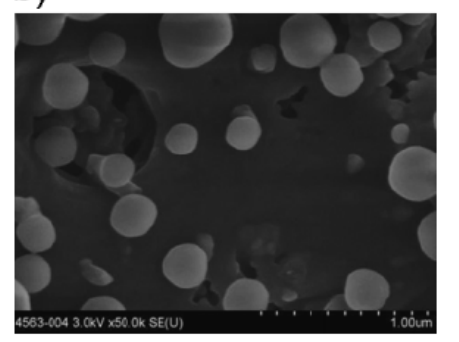

c)

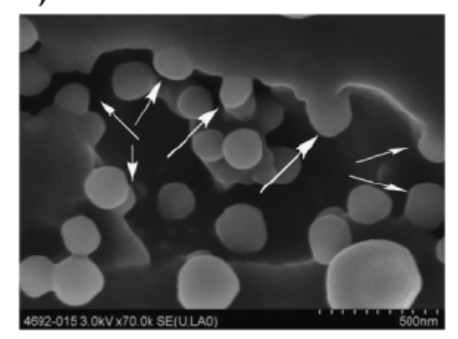

d)

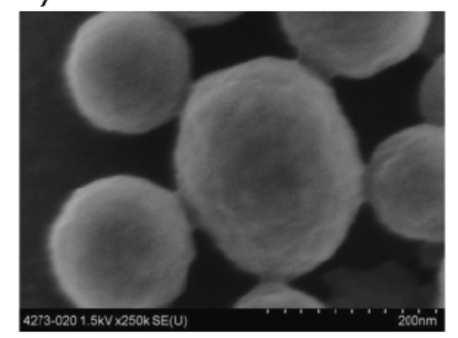

Figure 1. SEM images of the as-synthesized NAC material.

Figure 1 shows the low- and medium-magnification field- emission scanning electron microscopy (SEM) images of the as-synthesized composite carbon material (denoted as NAC). It is clear that the nanostructured carbon spheres are well-dispersed on the surface and pores of the AC host. In addition, it seems that the nanostructured carbon spheres grow from surface sites of the AC (see carbon spheres denoted by arrows in panel c of Fig. 1), indicating that the carbon nanospheres are sticking to the AC substrate well. A control ultrasonication experiment reveals that most of the carbon nanospheres remain on the AC host after ultrasonication treatment of NAC. This explicitly suggests that the as-synthesized NAC material features excellent integration characteristics. Most of the carbon spheres are approximately $100 \mathrm{~nm}$ in diameter, although a few carbon spheres have diameters either greater or less than this value. Figure 1d reveals the surface roughness of the nanostructured carbon spheres. Cross-sectional SEM images of NAC, as displayed in Figure S2 (Supporting Information), demonstrate that few carbon spheres are grown in the inner holes of the AC host, which can be attributed to the diffusion limitation of the glucose solution. The specific Brunauer-Emmett-Teller (BET) surface area and pore volume of the as- 
synthesized NAC material are $222 \mathrm{~m}^{2} \mathrm{~g}^{-1}$ and $0.147 \mathrm{~cm}^{3} \mathrm{~g}^{-1}$, respectively, which is much lower than the AC host values of $1506 \mathrm{~m}^{2} \mathrm{~g}^{-1}$ and $0.739 \mathrm{~cm}^{3} \mathrm{~g}^{-1}$, respectively. The Barrett-Joyner-Halenda (BJH) pore size distribution (as shown in Fig. S3, Supporting Information) shows that the volume of mesopores and micropores in the as-synthesized NAC material is much lower than that of AC or the AC host. Titirici et al. reported the replication and coating of silica templates by hydrothermal carbonization and found that hydrothermal carbon with different morphologies can be obtained by simply altering the polarity of the silica surface.[10] When the porous silica host achieves a suitable polarity, the hydrothermal carbon can fill its pores. A similar mechanism may also be present in the current work.

a)

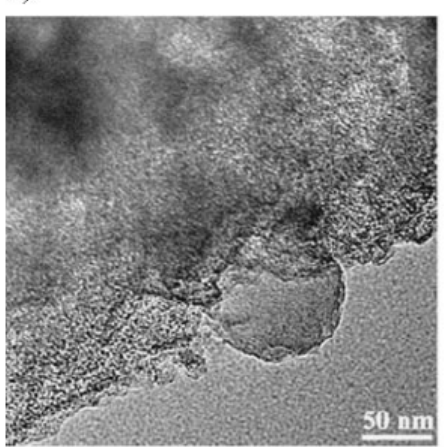

b)

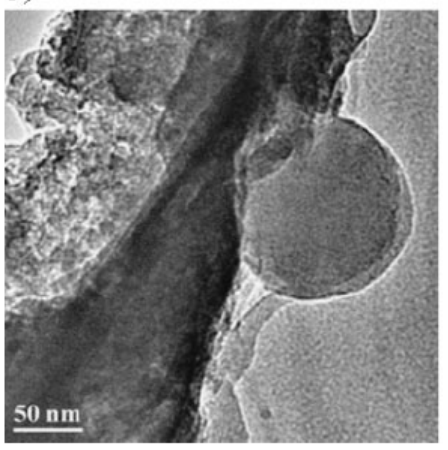

c)

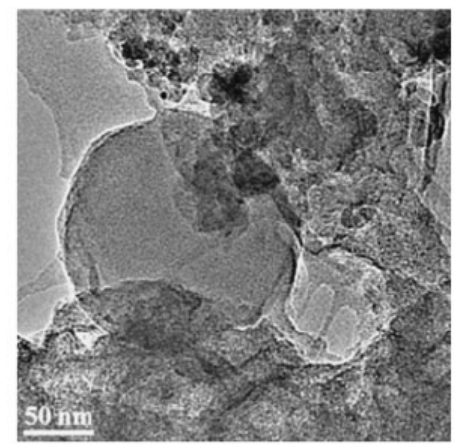

d)

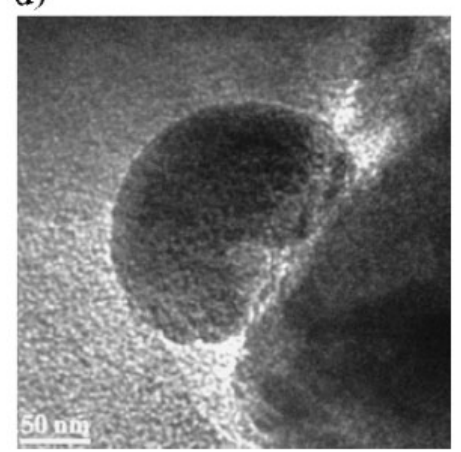

Figure 2. TEM images of the as-synthesized NAC material.

Transmission electron microscopy (TEM) and high-resolution TEM (HRTEM) were used to further characterize the microstructure of the as-synthesized composite carbon material. The TEM images, as displayed in Figure 2, demonstrate that nanostructured carbon spheres with various morphologies were generated under such autogenously high-pressure reaction conditions in the sealed autoclave. Carbon nanospheres are anchored onto or embedded in the AC surface matrix. Furthermore, perfect round-, elliptical-, or deformed-shaped carbon spheres were found, a finding that is distinctly different from the case of homogeneous formation of regular and uniform-sized carbon spheres when only an aqueous sugar solution was used.[11] This strongly indicates that the AC host plays an important role in the formation of the nanostructured carbon spheres, probably by providing heterogeneous nucleation sites to induce transformation of glucose molecules into carbon nanospheres with different kinds of morphologies. This inference can be partly substantiated by panel d in Figure 2. The HRTEM image of the interface between the carbon sphere and AC shows that the sphere has an amorphous structure (Fig. 3). This result suggests that the formed carbon spheres should have no influence on the X-ray diffraction (XRD) patterns of the AC host. This is consistent with the XRD results in Figure S4 of the Supporting Information; that is, no significant difference in the XRD patterns of the AC and NAC material is observed. On the other hand, note that short- range graphitic structure in the AC substrate is also clearly seen from the HRTEM image in Figure 3.

The effective anchoring of carbon nanospheres onto the AC host will concomitantly increase the surface functional groups of the newly structured activated carbon because the outer layer of carbon spheres is rich in $\mathrm{OH}$ groups and $\mathrm{C}=\mathrm{O}$ groups,[11] which are formed from non- or just partially dehydrated glucose molecules. Furthermore, some polymerized glucose units binding tightly to the surface of the AC host can also contribute to enhancing the oxygen-containing functional groups of the AC surface. In fact, the significantly increased amount of oxygen-containing functional groups on the NAC material has been 


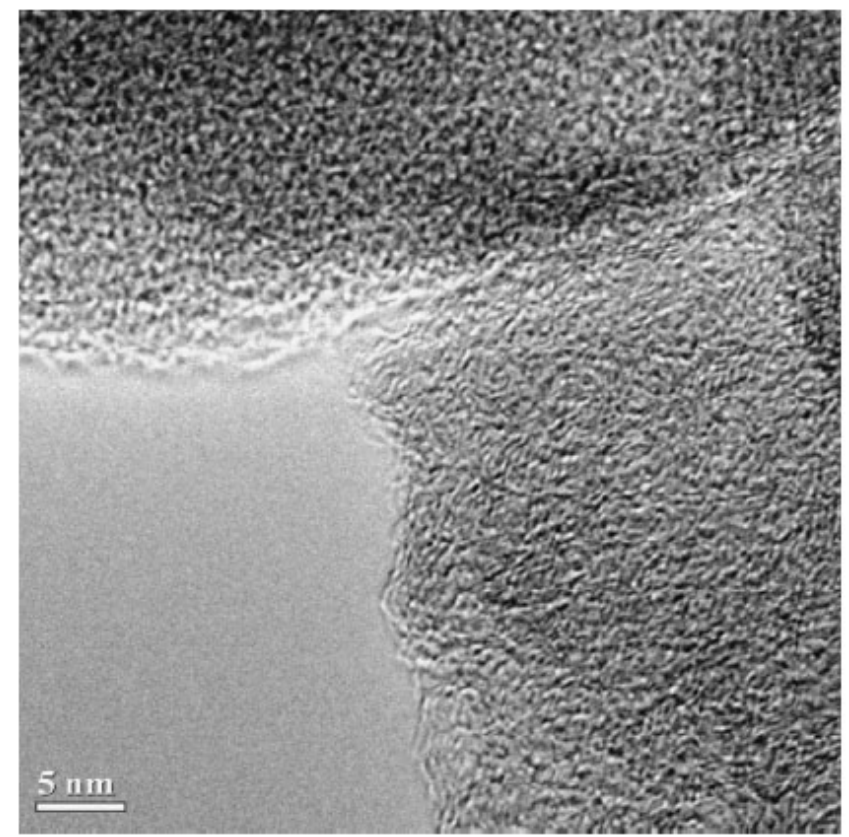

Figure 3. HRTEM image of the interface between a carbon sphere and the activated carbon substrate in the as-synthesized NAC material.

clearly substantiated by comparing the temperature program desorption (TPD) profiles of NAC with those of AC and AC-400, as shown in Figure 4a and b. Namely, the more remarkable intensities of the $\mathrm{CO}_{2}$ and $\mathrm{H}_{2} \mathrm{O}$ TPD profiles of NAC than those of AC and AC-400, performed under a helium atmosphere, clearly suggest that the as-synthesized NAC material contains more oxygen- containing surface functional groups. Further fitting the $\mathrm{CO}_{2}$ TPD profile leads to the assignment of each deconvolved peak to a specific type of surface oxygen group on the NAC material, as depicted in Figure 4c.[12] After the proper hydrothermal treatment of the AC host, the total amount of oxygen-containing functional groups, such as carbonyl, lactone, carboxylic, and anhydride groups, has been significantly increased.

Table 1. Quantitative XPS results of surface oxygen for the NAC material and the AC host. All values in at\%.

\begin{tabular}{|c|c|c|}
\hline Sample & O & C \\
\hline NAC & 22 & 78 \\
\hline AC-400 & 8.3 & 91.7 \\
\hline
\end{tabular}

X-ray photoelectron spectroscopy (XPS) analysis also reinforces the observation of more oxygencontaining functional groups on NAC than on the AC host. This is manifested by the greater O1s peak intensity for the as-synthesized NAC material than that for the AC host, as depicted in Figure 5a. From the areas of intensities in the O1s and C1s spectra, the quantification of surface composition for the NAC and AC host materials can be calculated. Results, showing the difference in surface functionalization of the materials, are summarized in Table 1 and suggest that the as-synthesized NAC material has a greater number of oxygen-containing surface functional groups than the AC host. Fitting of the XPS O1s spectrum gives us a detailed assignment of deconvolution peaks, as shown in Figure 5b. Four contributions can be primarily assigned to the O1s spectra.[12,13] The peak at around $531.3 \mathrm{eV}$ corresponds to carbonyl/C $=\mathrm{O}$ species; the peaks at around 533.4 and $532.1 \mathrm{eV}$ correspond to hydroxyl/C-OH and $\mathrm{C}-\mathrm{O}$ moieties, respectively. The small peak at around $535.8 \mathrm{eV}$ is assigned to adsorbed water. In addition, the presence of large amounts of residual $\mathrm{OH}$ groups on the NAC material is also qualitatively reflected by the increased intensity of bands in the range $1000-1300 \mathrm{~cm}^{-1}$ in the Fourier transform IR spectra (Fig. S5). The intensity of the $1710 \mathrm{~cm}^{-1}$ band is attributed to vibrations of the $\mathrm{C}=\mathrm{O}$ functional group in NAC; this band is also enhanced as compared to that in AC and the AC host. Thus far, it has been clearly corroborated that the as- synthesized NAC material has a significantly increased total amount of surface oxygen-containing functional groups as compared to that of the AC host.

As a result, the NAC material should have a notably enhanced adsorption capability for heavy metal ions than single-activated carbon, as the uptake of heavy metal ions from aqueous solution is generally assumed to be the main function of surface functional groups (mainly oxygen-containing functional groups), although other factors such as coordination with defective sites and van der Waals interactions 
a)

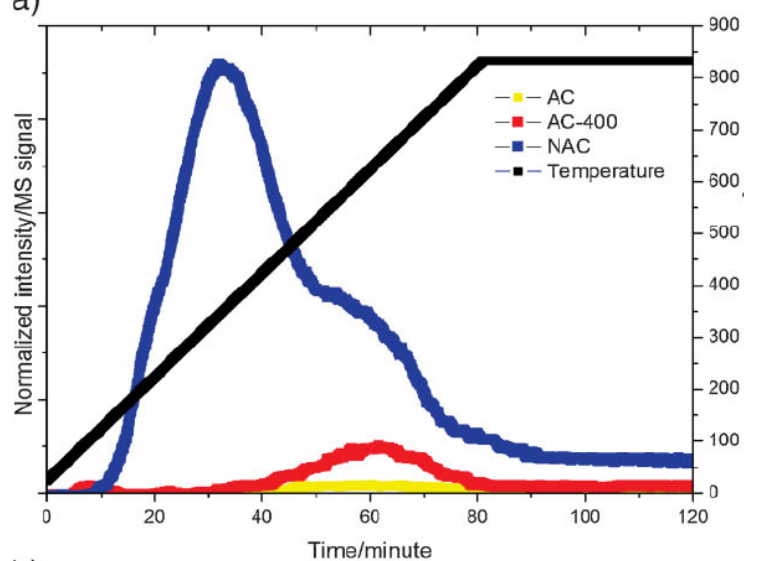

b)
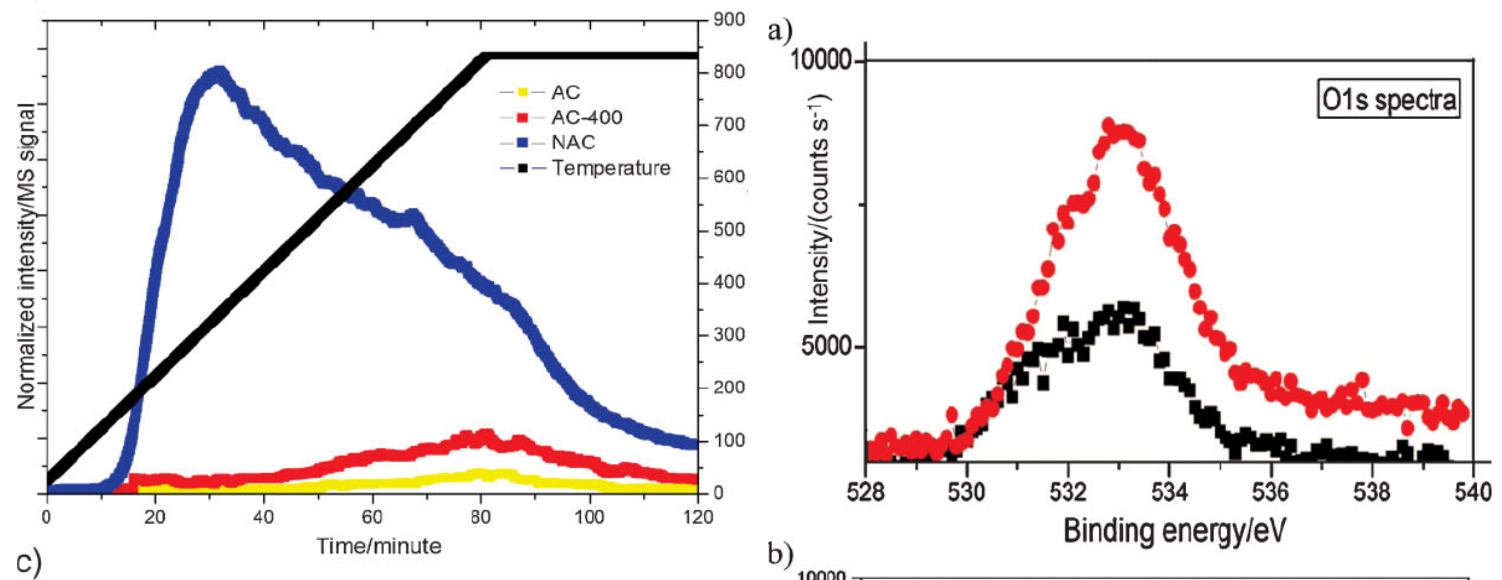

c)

b)

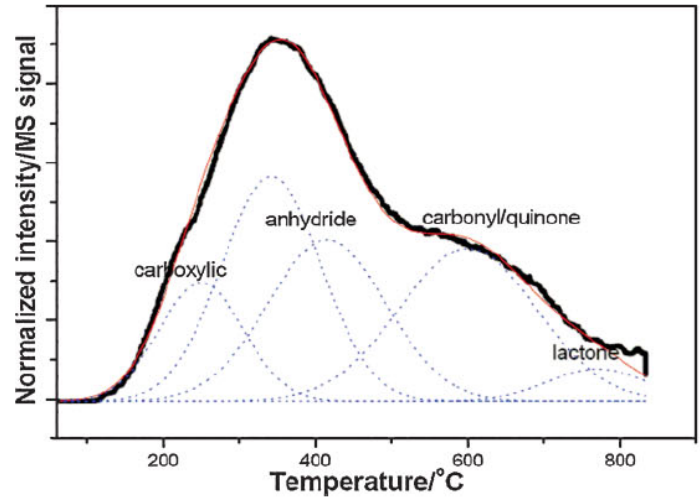

Figure 4. a) $\mathrm{CO}_{2}$ and b) $\mathrm{H}_{2} \mathrm{OTPD}$ profiles of the materials AC, AC-400, and NAC. c) Deconvolution of the $\mathrm{CO}_{2}$ TPD profile for the NAC material using a multiple Gaussian function.

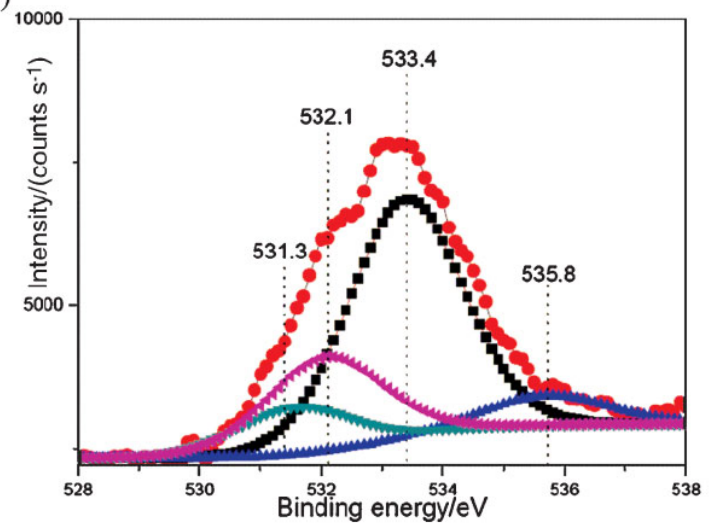

Figure 5. a) XPS O1s spectra of the as-synthesized NAC material (red line) and AC-400 (black line). b) Fitting of the XPS O1s spectrum for the NAC material

may also participate in the adsorption process.[5] This hypothesis has been confirmed by our preliminary adsorption experiments of anionic $\mathrm{CrO}_{4}{ }^{2-}$ and cationic $\mathrm{Fe}^{3+}$ species, which are two heavy metal ions typically found in waste water. Figures 6 and 7 display the adsorption isotherms of $\mathrm{CrO}_{4}{ }^{2-}$ and $\mathrm{Fe}^{3+}$, respectively. For example, based on the adsorption capacity per unit mass, the estimated maximumadsorption capacity of $\mathrm{CrO}_{4}{ }^{2-}$ for the NAC material is approximately $180 \mu \mathrm{mol} \mathrm{g}^{-1}$, which is much higher than the corresponding values of 32 and $60 \mu \mathrm{mol} \mathrm{g}^{-1}$ for AC and AC-400, respectively (Table 2). According to the data per unit surface area, the enhancement of adsorption capacity of $\mathrm{CrO}_{4}{ }^{2-}$ on NAC will become more significant as compared to that on AC and AC-400; the maximum adsorption capacity of $\mathrm{CrO}_{4}{ }^{2-}$ for the NAC material is about $0.81 \mu \mathrm{mol} \mathrm{m}{ }^{-2}$, remarkably higher than 0.030 and 0.040 $\mu \mathrm{mol} \mathrm{m}{ }^{-2}$, which correspond to AC and AC-400, respectively. This significant improvement of adsorption capability is mainly due to the increased total amount of surface functional groups (particularly $\mathrm{OH}$ groups) on NAC as compared to single AC because the adsorption of $\mathrm{CrO}_{4}{ }^{2-}$ is mainly by means of ion exchange with surface $\mathrm{OH}$ groups.[9] In this regard, we note that hierarchically structured carbon 
reported previously, consisting of carbon nanofibers nested and immobilized onto modified AC (ACsupported Fe catalyst), does not have the capability for adsorbing $\mathrm{CrO}_{4}{ }^{2-}$ as well as other heavy-metal ions.[9] This is due to the fact that, in such a high-temperature and reductive growth atmosphere as is provided by the CVD method, the original functional groups on the AC surface are deprived and it is also impossible to generate oxygen-containing functional groups during the growth of carbon fibers onto the AC host.[9] Without further chemical modifications, the hierarchically structuredcarbon as reported previously is not suitable for removing heavy-metal ions in aqueous solution.[9] In addition, it should be pointed out that a good carbon material for adsorption of heavy-metal ions should have both good adsorption capacity per unit mass and per unit surface area. Our NAC material shows considerably improved adsorption capacity of heavy metal ions based on both types of adsorption data, as compared to AC. Also, note that an analogous improvement of adsorption capability for cationic $\mathrm{Fe}^{3+}$ species has also been observed for our NAC material as compared to that for AC and AC-400, as is clearly shown in Figure 7. For instance, in aqueous $10 \mathrm{mM} \mathrm{Fe}^{3+}$ solution, the adsorption capacity per unit surface area for the as-synthesized NAC material is $6.76 \mu \mathrm{mol} \mathrm{m} \mathrm{m}^{-2}$, which is about ten times the value of the AC host (Table 2).
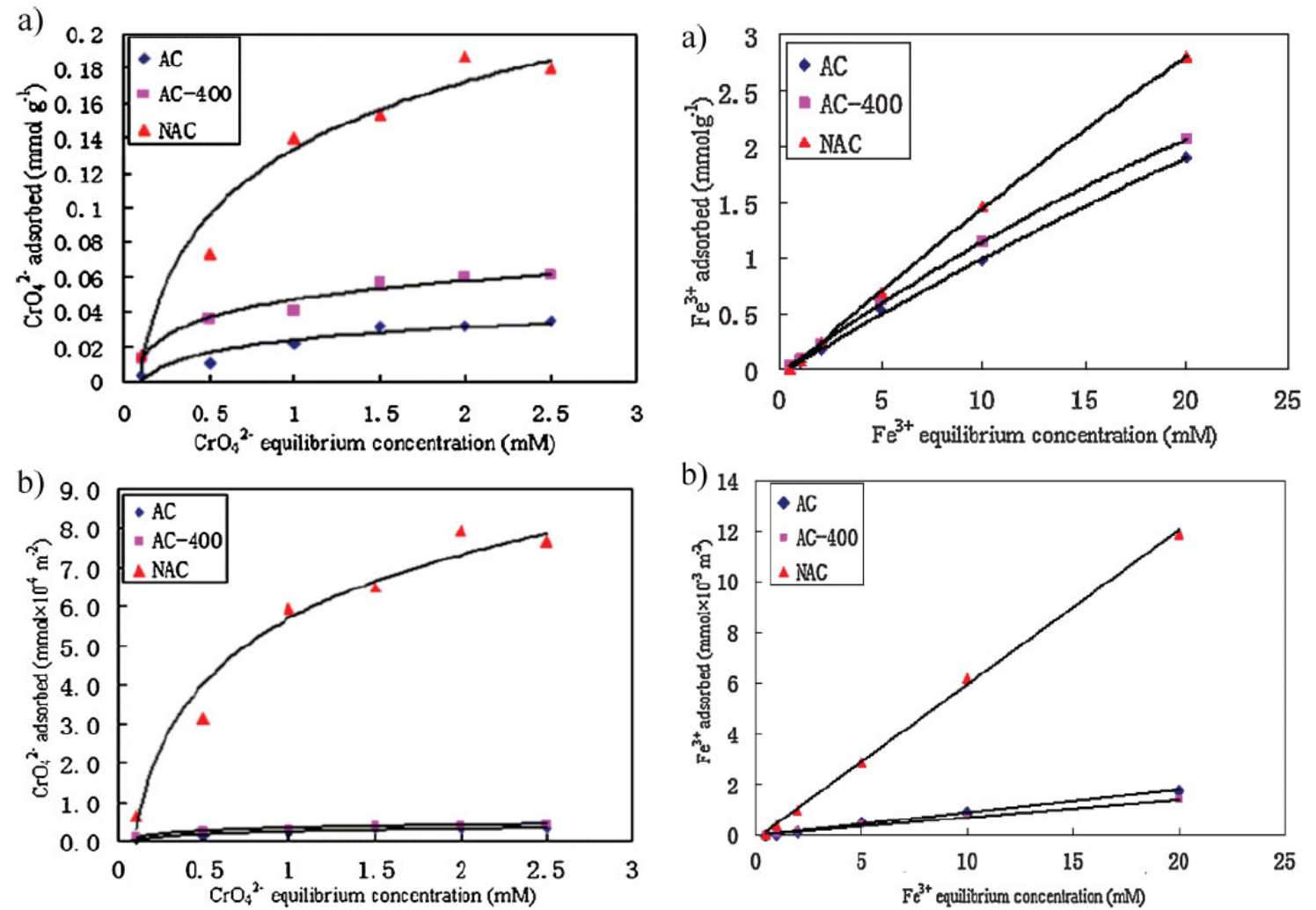

Figure 6. Adsorption isotherms of $\mathrm{CrO}_{4}{ }^{2-}$ in aqueous solution on AC, AC-400, and as-synthesized NAC. Adsorption capacity a) per unit mass and b) per unit surface area.

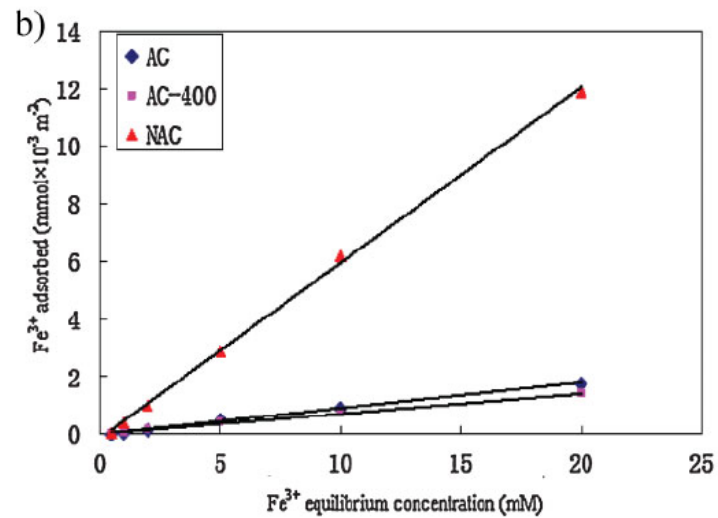

Figure 7. Adsorption isotherms of $\mathrm{Fe}^{3+}$ in aqueous solution on AC, AC-400, and as-synthesized NAC. Adsorption capacity a) per unit mass and b) per unit surface area.

In comparison to conventional methods for the surface modification of activated carbon,[5] it is clear that our method is effective, facile, and totally green because no strong acid, base, or harmful organic reagent is involved during the preparation of our hybrid NAC material. The increased oxygen-containing functional groups (e.g., $\mathrm{C}=\mathrm{O}$ and $\mathrm{OH}$ ) would be beneficial for the application of AC in catalytic reactions as the redox properties of AC are intimately related to the surface functional groups.[4a,14] On the other hand, the abundant $\mathrm{OH}$ and $\mathrm{C}=\mathrm{O}$ functional groups of the carbon spheres anchored on the $\mathrm{AC}$ surface can easily be subject to further modifications with heteroatoms that would give AC with other specific surface functionalities, as has been reported pre- viously.[10] In addition, the anchoring of carbon spheres onto the AC host increases the hydrophilicity of AC, and the functionalities can act as active sites to interact with metal particles.[15] In this respect, the as-obtained NAC material could also be used as an effective carbon support to prepare supported metal catalysts in heterogeneous catalysis. 


\section{Conclusions}

In summary, we have prepared a novel composite carbon material by anchoring carbon nanospheres onto the surface of activated carbon under mild conditions. The as-synthesized composite carbon material features a hierarchical structure, with a combination of macro- and nanometer-sized features, of the respective carbon components. In particular, the as- obtained composite carbon material has a remarkably enhanced capability to adsorb heavy metal ions from aqueous solution as compared to the single AC host. We have showed for the first time that nanoarchitecturing of activated carbon can be used as a new, facile, and green strategy for effective functionalization of the surface of activated carbon. Differing from conventional methods, no strong acids, bases, acute oxidants, or designed organic ligands were used. In addition to glucose, other kinds of polyhydric sugars, such as sucrose and fructose, can also be used for nanoarchitecturing of AC under similar hydrothermal conditions. Thus, this method opens a doorway for effective functionalization of the surface of activated carbon or other carbon materials.

Table 2. Adsorption of $\mathrm{CrO}_{4}{ }^{2-}$ and $\mathrm{Fe}^{3+}$ in aqueous solution by AC, AC-400, and as-synthesized NAC (data extracted from Figs. 6 and 7).

\begin{tabular}{|c|c|c|c|c|c|}
\hline Entry & $\mathrm{BET}\left[\mathrm{m}^{2} \mathrm{~g}^{-1}\right]$ & $\mathrm{CrO}_{4}{ }^{2-}[\mathrm{a}]\left[\mu \mathrm{mol} \mathrm{g}^{-1}\right]$ & $\mathrm{CrO}_{4}{ }^{2-}[\mathrm{a}]\left[\mu \mathrm{mol} \mathrm{m}^{-2}\right]$ & $\mathrm{Fe}^{3+}[\mathrm{b}]\left[\mu \mathrm{mol} \mathrm{g}^{-1}\right]$ & $\mathrm{Fe}^{3+}[\mathrm{b}]\left[\mu \mathrm{mol} \mathrm{m}^{-2}\right]$ \\
\hline AC & 1081 & 32 & 0.030 & 912 & 0.84 \\
\hline AC-400 & 1506 & 60 & 0.040 & 1105 & 0.73 \\
\hline NAC & 222 & 180 & 0.81 & 1501 & 6.76 \\
\hline
\end{tabular}

[a] The concentration of $\mathrm{CrO}_{4}{ }^{2-}$ is $2.5 \mathrm{~mm}$. [b] The concentration of $\mathrm{Fe}^{3+}$ is $10 \mathrm{~mm}$.

\section{Experimental}

The activated carbon was made from palm kernel shells, a waste product frompalmoil production. Palmkernel shells were carbonized in $\mathrm{N}_{2}$ at $350{ }^{\circ} \mathrm{C}$ and activated in steam at $600{ }^{\circ} \mathrm{C}$.Elemental analysis showed that the AC contained C (92.42 at\%), O (0.82 at\%), N (0.35 at\%), H (0.46 at\%), Si (0.71 at\%), and Fe (2.91 at\%). The AC host was obtained by calcination of $\mathrm{AC}$ at $400{ }^{\circ} \mathrm{C}$ for $4 \mathrm{~h}$ in air. The obtained composite carbon material (NAC) was synthesized as follows: the AC host ( $0.5 \mathrm{~g})$ was added to a glucose aqueous solution (30mL, $0.5 \mathrm{~mol} \mathrm{~L}^{-1}$ ) and stirred for $24 \mathrm{~h}$. Then, the mixtures were transferred to a $40 \mathrm{~mL}$ Teflon-sealed autoclave and maintained at $180-190^{\circ} \mathrm{C}$ for $3-6 \mathrm{~h}$ in the furnace. They were then cooled to room temperature. The products were completely washed with alcohol and distilled water and then dried in an oven for more than $8 \mathrm{~h}$.

Temperature program desorption (TPD) tests were carried out in a quartz reactor connected to a quadrupole mass spectrometer Prisma $200 \mathrm{M} 2)$. The sample $(20 \mathrm{mg})$ was loaded between two quartz wool plugs and placed in flowing helium at room temperature for $2 \mathrm{~h}$. After that, the temperature was raised to $830{ }^{\circ} \mathrm{C}$ with a ramp rate of $10^{\circ} \mathrm{C} \mathrm{min}^{-1}$, and the helium flow rate was maintained at $15 \mathrm{~mL} \mathrm{~min}{ }^{-1}$.

Powder XRD analysis was performed on an STOE STADI-P transmission diffractometer equipped with a focusing primary $\mathrm{Ge}(111)$ monochromator and a position-sensitive detector, using $\mathrm{Cu} \mathrm{K} \alpha 1$ radiation. Specific surface areas were measured by the BET method $\left(\mathrm{N}_{2}\right.$ physisorption at $\left.77 \mathrm{~K}\right)$ using an AUTOSORB-1-C physisorption/ chemisorption analyzer (Quantchrome).

XPS spectra were measured on beamline U49/2-PGM1 at BESSY in Berlin. SEM analysis was performed on a Hitachi S-4800 instrument at an acceleration electron voltage of $3 \mathrm{kV}$. TEM micrographs were taken on a Philips CM $200 \mathrm{LaB} 6$ at an acceleration electron voltage of $200 \mathrm{kV}$.

Adsorption isotherms of heavy-metal ions at room temperature were obtained by adding $10 \mathrm{mg}$ of AC, AC host, or NAC to a $2 \mathrm{~mL}$ Eppendorf cap, containing $1.5 \mathrm{~mL}$ of the appropriate adsorbate solution with different concentrations. The $\mathrm{pH}$ calculated for $\mathrm{CrO}_{4}{ }^{2-}$ (not adjusted with additional buffer) was around 8.0, which ensured that during the adsorption experiments the sole species adsorbed was bianionic $\mathrm{CrO}_{4}{ }^{2-}$. The $\mathrm{pH}$ for $\mathrm{Fe}^{3+}$ was around 1.8, adjusted with diluted $\mathrm{HCl}$ solution to prevent the hydrolysis of $\mathrm{Fe}^{3+}$ and its effect on the adsorption process. The suspensions were kept for 5 days to allow the adsorption of heavy-metal ions on the carbon reach the complete adsorption equilibrium. The concentration of $\mathrm{CrO}_{4}{ }^{2-}$ or $\mathrm{Fe}^{3+}$ after adsorption was measured by a Perkin-Elmer Lambda $650 \mathrm{UV}$-vis spectrometer.

\section{References}

[1] a) Nanostructured Carbon for Advanced Applications, (Eds: G. Benedek, P, Milani, V. G. Ralchenko ), Kluwer, Dordrecht, The Netherlands 2001. b) Carbon Nanomaterials, (Ed: Y. Gogotsi, ), CRC Press, Boca Raton, FL 2006. 
[2] a) F. Goettmann, A. Fischer, M. Antonietti, A. Thomas, Angew. Chem. Int. Ed. 2006, 45, 4467. b) Y. Hu, P. Adelhelm, B. Smarsly, S. Hore, M. Antonietti, J. Maier, Adv. Funct. Mater. 2007, 17, 1873. c) L. Zhi, J. Wu, J. Li, U. Kolb, K. Müllen, Angew. Chem. Int. Ed. 2005, 117, 2158. d) L. M. Dai, A. W. H.Mau, Adv. Mater. 2001, 13, 899. e) S. Scholz, P. J. Leech, B. C. Englert, W. Sommer, M. Weck, U. H. F. Bunz, Adv. Mater. 2005, 17, 1052. f) D. M. Guldi, G.M.A. Rahman, F. Zerbetto, M. Prato, Acc. Chem. Res. 2005, 38, 871. g) M. Terrones, Annu. Rev. Mater. Res. 2003, 33, 419. h) X. Lu, Z. Chen, Chem. Rev. 2005, 105, 3643. i) P. M. Ajayan, Chem. Rev. 1999, 99, 1787. j) S. Niyogi, M. A. Hamon, H. Hu, B. Zhao, P. Bhowmik, R. Sen,M. E. Itkis, C. R. Haddon, Acc. Chem. Res. 2002, 35, 1105.

[3] a) S. Frank, P. Poncharal, Z. L. Wang, W. A. Heer, Science 1998, 280, 1744. b) S. J. Tans, A. R. M. Verschueren, C. Dekker,Nature 1998, 393, 49. c) A. C. Dillon, K. M. Jones, T. A. Bekkedahl, C. H. Kiang, D. S. Bethune, M. J. Heben, Nature 1997, 386, 377.

[4] a) G. Mestl, N. I. Maksimova, N. Keller, V. V. Roddatis, R. Schlögl, Angew. Chem. Int. Ed. 2001, 40, 2066. b) Z. Zhu, D. S. Su,G.Weinberg, R. Schlögl, Nano Lett. 2004, 4, 2255. c) N. Keller,N. I.Maksimova,V.V. Roddatis, M. Schur, G. Mestl, Y. V. Butenko, V. L. Kuznetsov, R. Schlögl, Angew. Chem. Int. Ed. 2002, 41, 1885. d) J. J. Delgado, D. S. Su, G. Rebmann, N. Keller, A. Gajovic, R. Schlögl, J. Catal. 2006, 244, 126. e) Z. Zhu, D. S. Su, Y. Lu, R. Schlögl, G. Weinberg, Z. Liu, Adv. Mater. 2004, 16, 443. f) Y. S. Hu, P. Adelhelm, B. M. Smarsly, S. Hore, M. Antonietti, J. Maier, Adv. Funct. Mater. 2007, 17, 1873.

[5] a) J. García-Martín, R. López-Garzón, M. Luz Godino-Salido, M. Dolores Gutiérrez-Valero, P. ArranzMascarós, R. Cuesta, F. Carrasco-Marín, Langmuir 2005, 21, 6908. b) C. Moreno-Castilla, M. A. Ferro-Garcia, J. P. Joly, I. Bautista-Toledo, F. Carrasco-Marín, J. Rivera-Utrilla, Langmuir 1995, 11, 4386. c) P. J. M. Carrott, M. M. L. Carrott, J. M. V. Nabais, P. J. P. Ramalho, Carbon 1997, 35, 403. d) P. Vinke, M. van der Eijk, M. Verbree, A. F. Voskamp, H. Van Bekkum, Carbon 1994, 32, 675. e) Y. Liu, Y. Li, X. P. Yan, Adv. Funct. Mater. 2008, 18, 1.

[6] a) P. A. Brown, S. A. Gill, S. Allen, J. Water Res. 2000, 34, 3907. b) K. Csobán, M. Párkányi-Berka, P. Joó, P. Behra, Colloids Surf. A 1998, 141, 347. c) G. G. Jayson, J. A. Sangster, G. Thompson, M. C. Wilkinson, Carbon 1993, 31, 487. d) I. Bautista-Toledo, J. Rivera- Utrilla, M. A. Ferro-Garcia, C. Moreno-Castilla, Carbon 1994, 32, 93. e) B. E. Reed, S. Arunachalam, B. Thomas, Environ. Prog. 1994, 13, 60. f) Y. F. Jia, K. M. Thomas, Langmuir 2000, 16, 1114. g) Chemistry and Physics of Carbon, (Ed: L. R. Radovic, ), Vol. 27, Marcel Dekker, New York 2000, pp. 243-382.

[7] a) F. Schüth, Angew. Chem. Int. Ed. 2003, 42, 3604. b) A. H. Lu, F. Schüth, Adv. Mater. 2006, 18, 1793. c) R. Ryoo, S. H. Joo, S. Jun, J. Phys. Chem. B 1999, 103, 7743. d) S. Jun, S. H. Joo, R. Ryoo, M. Kruk, M. Jaroniec, Z. Liu, T. Ohsuna, O. Terasaki, J. Am. Chem. Soc. 2000, 122, 10 712. e) H. C. Foley, Microporous Mater. 1995, 4, 407. f) T. Kyotani, Carbon 2000, 38, 269. g) Y. Meng,D. Gu, F. Zhang, Y. Shi, H. Yang, Z. Li, C. Yu, B. Tu, D. Zhao, Angew. Chem. Int. Ed. 2005, 44, 7053. h) Z. Ma, T. Kyotani, A. Tomita, Chem. Commun. 2000, 2365.

[8] a) M. Hartmann, A. Vinu, G. Chandrasekar, Chem. Mater. 2005, 17, 829. b)M. B. Shiflett, H. C. Foley, Science 1999, 285, 1902. c) Y. D. Xia, R. Mokaya, Adv. Mater. 2004, 16, 886. d) J. Lee, J. Kim, T. Hyeon, Adv. Mater. 2006, 18, 2073. e) C. Liang, K. Hong, G. A. Guiochon, J. W. Mays, S. Dai, Angew. Chem. Int. Ed. $2004,43,5785$. f) S. Tanaka, N. Nishiyama, Y. Egashira, K. Ueyama, Chem. Commun. 2005, 2125.

[9] D. S Su, X. Chen, G. Weinberg, A. Klein-Hofmann, O. Timpe, S. B. Abd, Hamid, R. Schlögl, Angew. Chem. Int. Ed. 2005, 44, 5488.

[10] a) M. M. Titirici, A. Thomas, M. Antonietti, Adv. Funct. Mater. 2007, 17, 1010. b) M. M. Titirici, A. Thomas, M. Antonietti, J. Mater. Chem. 2007, 17, 3412.

[11] a) Q. Wang, H. Li, L. Q. Chen, X. J. Huang, Carbon 2001, 39, 2211. b) X. M. Sun, Y. D. Li, Angew. Chem. Int. Ed. 2004, 43, 597.

[12] J. Zhou, Z. Sui, J. Zhu, P. Li, D. Chen, Y. Dai, W. Yuan, Carbon 2007, 45, 785, and references therein.

[13] a) X. Liu, D. S. Su, R. Schlögl, Carbon 2008, 46, 547. b) E. Desimoni, G. I. Casella, A. Morone, A. M. Salvi, Surf. Interface Anal. 1990, 15, 627.

[14] a) C. Moreno-Castilla, F. Carrasco-Marin, C. Parejo-Perez, M. V. Lopez-Ramon, Carbon 2001, 39, 869. b) C. A. Leon y Leon, L. R. Radovic, Chem. Phys. Carbon. c) F. Carrasco-Marin, A. Mueden, C. Moreno-Castilla, J. Phy. Chem. B 1998, 102, 9239. d) H. P. Boehm, G. Mair, T. Stoehr, A. R. De Rincon, B. Tereczki, Fuel 1984, 63, 1061.

[15] a) A. Guha, W. Lu, T. A. Zawodzinski, Jr, D. A. Schiraldi, Carbon 2007, 45, 1506. b) M. C. Román-Martínez, D. Cazorla-Amoros, A. Linares-Solano, C. Salinas-Martínez de Lecca, Curr. Top. Catal. 1997, 1, 17. c) M. C. Román-Martínez, J. A. M. Díaz-Aunón, C. Salinas- Martínez de Lecca, C. H. Alper, J. Mol. Catal. A 2004, 213, 177. d) E. Auer, A. Freund, T. Tacke, J. Pietsch, Appl. Catal. A 1998, 173, 259 